\title{
Estimating traffic disruption patterns with volunteer geographic information
}

\author{
Bright, Jonathan ${ }^{\mathrm{a}}$; Camargo, Chico ${ }^{\mathrm{a}}$; Hale, Scott ${ }^{\mathrm{a}}$; McNeill, Graham ${ }^{\mathrm{a}}$ and Raman, \\ Sridhar ${ }^{\text {b }}$ \\ ${ }^{\mathrm{a}}$ Oxford Internet Institute, University of Oxford, UK, ${ }^{\mathrm{b}}$ Oxford Brookes University, Oxford, \\ UK.
}

\begin{abstract}
Accurate understanding and forecasting of traffic conditions is a key contemporary problem for local policymakers. Road networks are increasingly congested, yet data on usage patterns is often scarce or expensive to obtain, meaning that informed policy decision-making is difficult. This paper explores the extent to which traffic disruption can be estimated from static features of the volunteer geographic information site OpenStreetMap [OSM]. Kernel Density Estimates of OSM features are used as predictors for a linear regression of counts of traffic incidents at 6,500 separate points within the Oxfordshire road traffic network. For highly granular points of just $10 \mathrm{~m}^{2}$, it is shown that more than half of variation in traffic outcomes can be explained with these static features alone. Furthermore, use of OSM's granular point of interest data improves considerably on more aggregate categories which are typically used in studies of transportation and land use. Although the estimations are by no means perfect, they offer a good baseline model considering the data is free to obtain and easy to process.
\end{abstract}

Keywords: traffic networks; land use; social media; open data.

This project was supported by funding from InnovateUK under grant number 52277393176, the NERC under grant number NE/N00728X/1, and the Lloyd's Register Foundation. 